\title{
Meibomian Gland Morphology: The Influence of Structural Variations on Gland Function and Ocular Surface Parameters
}

\author{
Clara Llorens-Quintana, PhD, * Laura Rico-del-Viejo, PhD, $†$ Piotr Syga, PhD, $\neq$ \\ David Madrid-Costa, PhD, $\dagger$ and D. Robert Iskander, PhD, DSc*
}

Purpose: To objectively and quantitatively characterize meibomian gland morphology and to investigate the influence of morphological variations on gland function and ocular surface and tear film parameters.

Methods: One hundred fifty subjects were enrolled. The examinations included tear osmolarity, tear meniscus height, bulbar conjunctival hyperemia, noninvasive tear film breakup time, lid margin thickness, foam secretion, meibomian gland expressibility, count of functioning glands, corneal and conjunctival staining, fluorescein breakup time, lid wiper epitheliopathy, and Schirmer test. Patient symptoms were assessed using the Ocular Surface Disease Index questionnaire. Images from noncontact meibography were analyzed using an automated method that objectively estimates dropout area, number of glands, gland length and width, and gland irregularity.

Results: Gland irregularity highly correlated with dropout area $(r=$ $-0.4, P<0.001)$ and showed significant partial correlations with fluorescein breakup time $(r=0.162, P=0.049)$ and the Ocular Surface Disease Index questionnaire $(r=-0.250, P=0.002)$ Subjects with dropout area $<32 \%$ were divided into 2 groups: high and low irregularity. Gland expressibility was statistically significantly different between the 2 groups $(U=319.5, P=0.006)$. In the high irregularity group, gland irregularity correlated with the

Received for publication March 27, 2019; revision received July 15, 2019; accepted July 23, 2019.

From the *Department of Biomedical Engineering, Faculty of Fundamental Problems of Technology, Wrocław University of Science and Technology, Wroclaw, Poland; †Department of Optometry and Vision, Faculty of Optics and Optometry, Complutense University of Madrid, Madrid, Spain; and $\ddagger$ Department of Computer Science, Faculty of Fundamental Problems of Technology, Wrocław University of Science and Technology, Wroclaw, Poland.

Supported by the European Union's Horizon 2020 research and innovation program under the Marie Skłodowska-Curie Actions (grant number 642760).

The authors have no conflicts of interest to disclose.

C. Llorens-Quintana and L. Rico-del-Viejo have contributed equally to this study.

Correspondence: Clara Llorens-Quintana, PhD, Department of Biomedical Engineering, Faculty of Fundamental Problems of Technology, Wrocław University of Science and Technology, Wybrzeze Wyspianskiego 27, Wroclaw, 50-370, Poland (e-mail: Clara.1lorens.quintana@pwr.edu.pl).

Copyright ( 2019 Wolters Kluwer Health, Inc. All rights reserved.
Schirmer test $(r=0.530, P=0.001)$ and corneal fluorescein staining $(r=-0.377, P=0.021)$.

Conclusions: Automated morphological analysis of meibomian gland structure provides additional quantitative and objective information regarding gland morphology. The link between dropout area and gland function is not clear. Assessment of gland irregularity might better predict gland function and its effects on ocular surface and tear film parameters.

Key Words: meibomian glands, meibography, meibomian glands morphology, objective medical image analysis, morphological image analysis

(Cornea 2019;00:1-7)

M eibomian glands play an important role in maintaining the ocular surface equilibrium because they secrete the lipids that form the main part of the tear film lipid layer. The importance of the tear film lipid layer and its impact on tear film homeostasis is well known. ${ }^{1,2}$ The assessment of meibomian gland morphology is currently receiving much attention because of the impact of meibomian gland dysfunction (MGD) in dry eye disease worldwide. ${ }^{2,3}$ Since the introduction of noncontact infrared meibography in the clinical setting, ${ }^{4}$ both researchers and eye care practitioners have been able to assess the structure of meibomian glands in an easy and patient-friendly manner, leading to improvement in the diagnosis of MGD and dry eye disease. Infrared meibography allows the observation of meibomian gland structure and abnormalities such as loss, shortening, dilation, or tortuosity of the glands. ${ }^{5}$ Meibomian gland loss or dropout has been previously studied as an indicator of MGD and correlated with its severity. ${ }^{6}$ In addition, although less reported, meibomian gland length and width have also been studied. ${ }^{7,8}$ Earlier studies reported subjective grading of meibomian gland morphological features. However, in the past few years, the assessment of meibomian gland morphology is advancing toward an objective and automated approach because of its numerous benefits. ${ }^{9-12}$ It has been suggested that objective and automatic methods would provide more consistent and reliable results. ${ }^{5}$ Also, in addition to meibomian gland dropout area, automated methods can provide a more complete morphological 
characterization of the glands (such as gland length, width, shape, contour or tortuosity), leading to improvement in the diagnosis of MGD. ${ }^{13-15}$

Only few automated methods, which objectively analyze meibomian gland structure, have been proposed. However, these methods provide limited information regarding the relationship between objective meibomian gland morphological parameters and ocular surface and tear film parameters. ${ }^{9-12}$ For this reason, the purpose of this study is to investigate the relationship between objective meibomian gland morphological features, obtained using an automated method, and meibomian gland function, tear film quality, and ocular surface parameters to ascertain what is the impact of meibomian gland morphological alterations on clinically relevant ocular surface parameters.

\section{MATERIALS AND METHODS}

\section{Subjects}

A total of 150 subjects ( 80 women and 70 men) were included in this study. The study protocol was reviewed and approved by the Ethics Committee of San Carlos University Hospital (Madrid, Spain) and adhered to the tenets of the Declaration of Helsinki. Mean age \pm SD of the subjects was of $42 \pm 17$ years (19-88 yrs). Forty-seven (31\%) subjects were current contact lens wearers, of which 2 were daily soft contact lens wearers, 44 were either monthly or biweekly soft contact lens wearers, and 1 was a rigid gas permeable contact lens wearer. Subjects were recruited for the study from the general public through advertising. Written informed consent was obtained from all participants after explaining the purpose and the possible adverse effects of the study. The clinical examination was carried out in the Faculty of Optics and Optometry of the Complutense University of Madrid between October 1, 2016, and September 30, 2017. Inclusion criteria included being 18 years old or older, being able to complete the questionnaires, understanding the procedures, and obtaining evaluable meibography images of the upper eyelid. Exclusion criteria included history of any active ocular disease different from dry eye disease and meibomian gland dysfunction/disease (MGD) (eg, corneal ulcers, herpes simplex, keratitis), any uncontrolled severe systemic disease that might have affected the eye (eg, Sjögren syndrome, diabetes type II, dermatological disease), or any ocular surgery or trauma that could affect the tear film or produce any eyelid margin abnormality. No exclusion was made regarding the state of subjects' meibomian glands because the aim was to include subjects with varying levels of meibomian gland health. Contact lens wearers were accepted, but they were required not to use their contact lenses within a week before the clinical examination.

\section{Ocular Surface Evaluation}

Both dry eye symptomatology and ocular surface characteristics were assessed in a single visit for the right eye of each participant. The clinical examination was performed in the same room with a mean \pm SD room temperature of $22^{\circ} \mathrm{C} \pm 2{ }^{\circ} \mathrm{C}$ and a mean $\pm \mathrm{SD}$ relative humidity of $30 \% \pm 5 \%$. All ocular measurements were accomplished by the same investigator and conducted in ascending order of invasiveness to minimize the effect of the previous measurement. Measurements included dry eye symptomatology, tear film osmolarity, tear meniscus height, bulbar conjunctival hyperemia, noninvasive tear film breakup time, slit-lamp examination (ocular surface integrity and eyelid features), and Schirmer test.

Dry eye subjective symptomatology was assessed with the Ocular Surface Disease Index (OSDI) questionnaire. ${ }^{16}$

Tear film osmolarity was assessed using the TearLab osmometer (TearLab, San Diego, CA) according to the manufacturer's instructions. The participant was seated with the chin tilted upward and eyes directed toward the ceiling. The device pen was positioned just above the lower eyelid, in the tear meniscus, avoiding contact with the globe during the measurement. Afterward, the device was returned to the electronic base to obtain the osmolarity reading. One measurement of each eye was performed, but only the reading of the right eye was used for analysis.

Noninvasive measurements were performed using the Keratograph 5M (K5M; Oculus Optikgerate, Wetzlar, Germany). ${ }^{17}$ Three measurements of the tear meniscus height, bulbar conjunctival hyperemia, the first tear film breakup time, and the average tear film breakup time incidents were obtained automatically using the software provided with the $\mathrm{K} 5 \mathrm{M}$. Tear meniscus height was measured perpendicularly to the lid margin at the central point relative to the pupil center using an integrated ruler. Ocular redness was automatically evaluated with $\mathrm{K} 5 \mathrm{M}$ software by scanning the image of the exposed bulbar conjunctiva. The software generates a score based on the ratio between blood vessels area and total bulbar conjunctiva area.

Slit-lamp examination of the ocular surface was performed under diffuse illumination using $\times 10$ to $\times 16$ (Topcon SL-D4, Tokyo, Japan). Corneal staining was assessed by instilling fluorescein using a fluorescein strip (Tiedra Laboratories, Madrid, Spain) that was previously wetted with 1 drop of saline solution (Saline solution; Avizor, Madrid, Spain) and applied to the eye. Then, the staining was graded using the Oxford scoring scheme. ${ }^{18}$ Fluorescein breakup time was measured 3 times with a stopwatch and was averaged for the subsequent analysis. In addition, conjunctival staining was assessed using a lissamine green strip (Tiedra Laboratories) previously wetted with 1 drop of saline solution and graded using the Oxford scoring scheme.

Eyelid features such as lid margin thickness, expressibility, foam secretion, number of functional meibomian glands, and lid wiper epitheliopathy were evaluated with the slit lamp. Eyelid margin thickness was assessed on a scale from 1 to 5 , where 1 to $2=$ thin; $3=$ normal; and 4 to $5=$ thick. Foam secretion was categorized as 0 (absent) or 1 (present). Expressibility from the 8 central meibomian glands was assessed on a scale from 0 to 3 , where $0=$ clear meibum readily expressed; 1 = cloudy meibum expressed with mild pressure; 2 = cloudy meibum expressed with more than moderate pressure; and $3=$ meibum could not be expressed 
even with strong pressure. The number of functional meibomian glands was assessed on a scale from 0 to 3 , where $0=$ more than 5 glands expressible; $1=3$ to 4 glands expressible; $2=1$ to 2 glands expressible; and $3=$ no glands expressible. Lid wiper epitheliopathy was assessed using a combination of fluorescein and lissamine green (Korb Protocol B), where the higher of the final fluorescein or lissamine green staining score is used as the lid wiper epitheliopathy severity grade (0 $=$ absent, $1=$ mild, 2 = moderate, and $3=$ severe) ${ }^{19}$

The modified Schirmer I test was performed with topical anesthesia (Colirio Anestésico Doble, Spain) and read at 5 minutes with a cutoff point of $10 \mathrm{~mm} .{ }^{20}$ The Schirmer strip was placed in the lower conjunctival sac at the junction of the lateral and middle thirds, and after 5 minutes, the length of wetting was recorded. Participants were seated, and they were asked to close the eyes during the test.

\section{Infrared Meibography and Data Analysis}

Infrared meibography images of the everted upper eyelid were acquired with the $\mathrm{K} 5 \mathrm{M}$ infrared camera (before the Schirmer test was performed). Bit map images were exported and analyzed offline using a recently proposed automated algorithm that provides a morphometric characterization of the meibomian gland structure. A brief description of the main steps of the method is provided below. For more technical details, please refer to Llorens-Quintana et al. ${ }^{21}$

The algorithm used is based on different image processing techniques, and it is divided into 3 main consecutive and automated steps: 1) selection of the area of analysis which is the tarsal conjunctiva, 2) isolation of the individual meibomian glands, and 3) extraction of meibomian gland morphological features (ie, dropout area, gland length, gland width, number of glands, and gland irregularity). These morphological features allow an objective and quantitative description of the general condition of meibomian glands and a more specific characterization of the gland structure.

The dropout area is calculated as the percentage of the total analyzed area (ie, the exposed tarsal conjunctiva) that has gland dropout.

To estimate the length and width of the glands, each gland is fitted with an ellipse having the same normalized central moment of the gland. Then, the length and width of the gland are approximated to the length of the major and minor axis of the fitted ellipse, respectively. Finally, the mean length and width of all the exposed glands of the tarsal conjunctiva is computed and reported.

The number of glands corresponds to the number of detected and analyzed glands per eyelid.

Gland irregularity is described regarding shape dissimilarity of each gland from a standardized regular gland. To determine the shape of the standardized gland, the normalized edge coordinates of 300 regular glands were determined and averaged, shaping the "standard gland." Then, the shape of each detected gland is superposed to the shape of the "standard gland," and gland irregularity is defined as their differential area. To provide a more understandable measure, this differential area is expressed as a percentage of the total area of the "regular gland" and it can be understood as the amount of gland being out of the regular boundaries. Thus, the higher the extent of the differential area, the greater the irregularity of the gland. Finally, to provide a global measure of irregularity per eyelid, the mean irregularity of all the glands in the exposed tarsal conjunctiva is computed and reported.

All images were processed in a batch mode with no user input, and the results were automatically saved. However, manual selection of the exposed tarsal conjunctiva was allowed if the algorithm did not properly identify it. This happened in 9 of 150 images, in 6 of which there was an acquisition problem and in the remaining 3 there was a failure in the preprocessing steps. In all cases, the following glands isolation and features extraction steps were performed automatically.

\section{Statistical Analysis}

Mean \pm SD (only for continuous measures), median (IQR), and range of values have been used to describe the group values for the clinical tests and objective parameters. Because data were not normally distributed, nonparametric tests have been used for statistical testing. To assess the influence of meibomian gland morphology in meibomian gland function and ocular surface, the correlation among the objective morphological parameters of meibomian glands and clinical tests were assessed with 2-tailed Spearman $r$. Differences among groups with different irregularity levels have been assessed with a Mann-Whitney $U$ test for independent samples. For all statistical tests, a significance level of $P<$ 0.05 was set.

\section{RESULTS}

Raw infrared images of meibomian glands from the upper eyelid were analyzed in an automated fashion to extract the objective morphological features. Descriptive values of the objective features and clinical signs and symptoms for all participants are shown in Table 1.

Spearman correlations between objective features and clinical signs and symptoms were assessed to study the possible effects of meibomian gland morphology on meibomian gland function and ocular surface health.

Table 2 shows those correlations that were statistically significant. They will be discussed later.

As can be seen, gland irregularity has the opposite trend to what could be expected, meaning that more irregularity would entail better ocular surface conditions. However, this should not be misinterpreted. Gland irregularity is inversely correlated with the dropout area (Spearman $r=-0.4, P<$ $0.001)$. In general, it is more likely that longer glands, which usually entail less dropout area, could have more irregularity compared with short glands. Figure 1 shows 3 meibography images with different dropout areas (ie $5 \%, 20 \%$, and $61 \%$ for Figures $1 \mathrm{~A}, 1 \mathrm{~B}$, and $1 \mathrm{C}$, respectively) and different irregularity levels. It can be noticed that in Figure $1 \mathrm{~A}$, where the glands are long, they are more irregular than those shown in Figure 1C, where the short glands have less irregularity. In general, the longer the glands are the more likely they are 
TABLE 1. Descriptive Statistics for the Extracted Objective Features, Clinical Tests, and Subjective Questionnaires

\begin{tabular}{|c|c|c|c|}
\hline & Mean \pm SD & Median (IQR) & Range \\
\hline \multicolumn{4}{|l|}{ Objective features } \\
\hline DOA $(\%)$ & $21.6 \pm 15.3$ & $19.5(23)$ & $0-75$ \\
\hline $\begin{array}{l}\text { Mean gland } \\
\text { length (mm) }\end{array}$ & $3.3 \pm 0.6$ & $3.3(0.9)$ & $1.82-4.59$ \\
\hline $\begin{array}{l}\text { Mean gland } \\
\text { width }(\mathrm{mm})\end{array}$ & $0.5 \pm 0.1$ & $0.5(0.1)$ & $0.3-0.7$ \\
\hline Number of glands & $17 \pm 6$ & $18(7)$ & $3-35$ \\
\hline Irregularity $(\%)$ & $21.6 \pm 9.4$ & $19.8(12.5)$ & $0-54.8$ \\
\hline \multicolumn{4}{|l|}{ Clinical tests } \\
\hline Osmolarity $(\mathrm{mOsm} / \mathrm{L})$ & $310 \pm 18$ & $306(20)$ & $283-385$ \\
\hline TMH (mm) & $0.26 \pm 0.08$ & $0.25(0.09)$ & $0.13-0.53$ \\
\hline Bulbar redness & $1.11 \pm 0.50$ & $1.03(0.70)$ & $0.0-3.33$ \\
\hline NIKBUT-fr (s) & $8.9 \pm 5.3$ & $7.0(6.8)$ & $1.9-23.1$ \\
\hline NIKBUT-avg (s) & $12.0 \pm 5.2$ & $11.5(8.0)$ & $2.1-25$ \\
\hline $\begin{array}{l}\text { Lid margin thickness } \\
\quad \text { (5-grade scale) }\end{array}$ & - & $4(1)$ & $2-5$ \\
\hline $\begin{array}{l}\text { Foam secretion } \\
\text { (2-grade scale) }\end{array}$ & - & $0(1)$ & $0-1$ \\
\hline $\begin{array}{l}\text { Expressibility } \\
\quad \text { (4-grade scale) }\end{array}$ & - & $1(1)$ & $0-3$ \\
\hline $\begin{array}{l}\text { Number of gland } \\
\text { functioning } \\
\text { (4-grade scale) }\end{array}$ & - & $0(1)$ & $0-2$ \\
\hline $\begin{array}{l}\text { Corneal staining } \\
\text { (5-grade scale) }\end{array}$ & - & $1(1)$ & $0-4$ \\
\hline FBUT (s) & $4.4 \pm 2.1$ & $3.7(2.0)$ & $1.0-16.7$ \\
\hline $\begin{array}{l}\text { Conjunctival staining } \\
\text { (5-grade scale) }\end{array}$ & - & $1(1)$ & $0-4$ \\
\hline LWE (4-grade scale) & - & $1(0)$ & $0-3$ \\
\hline Schirmer (mm) & $12.0 \pm 7.2$ & $11.0(7.0)$ & $0-35.0$ \\
\hline \multicolumn{4}{|l|}{ Subjective questionnaire } \\
\hline OSDI & $16.7 \pm 15.0$ & $13.1(17.1)$ & $2.1-75$ \\
\hline
\end{tabular}

DOA, dropout area; FBUT, fluorescein breakup time; LWE, lid wiper epitheliopathy; NIKBUT-avg, average noninvasive tear film breakup time; NIKBUT-fr, first noninvasive tear film breakup time; TMH, tear meniscus height.

irregular. This can be noted in Figure 1B where the arrow points to a long and irregular gland and the asterisk demarcates a group of short glands in which it is more difficult to find high levels of irregularity.

Accordingly, to avoid the potentially misleading results of the Spearman correlation, partial correlations between gland irregularity and clinical signs and symptoms were computed, setting the dropout area as the control variable. Irregularity showed statistically significant partial correlations with the fluorescein breakup time $(r=0.162, P=0.049)$ and OSDI $(r=-0.250, P=0.002)$. However, it seems that measuring gland irregularity in those subjects who have a relatively large dropout area does not provide useful information about the condition of the meibomian glands. As discussed above, shortened glands are rarely tortuous. Thus, the study of gland irregularity was narrowed to subjects with dropout area $\leq 32 \%$. This threshold was chosen because it divides the sample into 2 groups, according to the amount of dropout area, with minimal intraclass variance using the
TABLE 2. Statistically Significant Correlations Between Objective MG Morphology Features and Clinical Tests

\begin{tabular}{lcc}
\hline & Spearman Correlation & $\boldsymbol{P}$ \\
\hline DOA-TMH & 0.247 & 0.002 \\
DOA-bulbar redness & 0.188 & 0.022 \\
DOA-conjunctival staining & 0.193 & 0.018 \\
Mean gland length-Lid margin thickness & -0.163 & 0.046 \\
Mean gland length-FBUT & 0.175 & 0.032 \\
Mean gland width-Lid margin thickness & -0.162 & 0.048 \\
Mean gland width-Foam secretion & -0.177 & 0.031 \\
Mean gland width-FBUT & 0.176 & 0.031 \\
Mean gland width-Conjunctival staining & -0.179 & 0.029 \\
Irregularity-FBUT & 0.254 & 0.013 \\
Irregularity-conjunctival staining & -0.227 & 0.027 \\
Irregularity-OSDI & -0.264 & 0.010 \\
\hline
\end{tabular}

DOA, dropout are; FBUT, fluorescein breakup time; TMH, tear meniscus height.

$\mathrm{Otsu}^{22}$ method. Accordingly, 110 of 150 subjects had a dropout area $\leq 32 \%$. This group was composed of 59 women and 51 men with an age range from 19 to 80 years and a mean \pm SD age of $40 \pm 17$ years. Mean \pm SD irregularity for this group was $21.16 \% \pm 8.34 \%$. Figure 2 shows the distribution of gland irregularity for the reduced group. The reduced group was further divided into 2 subgroups: 1) subjects with low irregularity and 2) subjects with high irregularity. Data partitioning was performed using the $\mathrm{k}$-means clustering algorithm (with $\mathrm{k}=2$ ), an iterative algorithm which assigns each observation to one of the 2 groups so the squared Euclidean distance of each point to each group centroid is minimal. Accordingly, 72 subjects formed the group of low irregularity and 38 subjects were included in the high irregularity group. Mean \pm SD gland irregularity was $16.35 \% \pm 4.62 \%$ and $30.26 \% \pm 5.82 \%$ for the low and high irregularity groups, respectively. The threshold for this division was an irregularity of $23.2 \%$.

Table 3 shows the values of the clinical parameters for the high and low irregularity groups. The Mann-Whitney $U$ test revealed statistically significant differences in gland expressibility among both groups $(U=319.5, P=0.006)$. In the high irregularity group, Schirmer test results correlated with irregularity $(r=0.530, P=0.001)$, and there was inverse correlation between corneal staining and irregularity $(r=$ $-0.377, P=0.021$ ) after controlling for the dropout area variable. In that group, gland expressibility did not correlate with gland irregularity $(r=-0.149, P=0.380)$. In addition, gland irregularity showed no statistically significant correlation with any of the other clinical measures.

\section{DISCUSSION}

In this study, the relationship between different meibomian gland morphology descriptors and clinical parameters of gland function, tear film quality, and ocular surface integrity has been evaluated using a fully automated analysis. ${ }^{21}$

Several studies have subjectively evaluated the relationship between meibomian gland dropout and meibomian gland 
FIGURE 1. Example of 3 infrared meibography images with a dropout area of $5 \%(A), 20 \%(B)$, and $61 \%$ (C). It can be seen that shortened glands have low irregularity, whereas higher levels of irregularity might be found in longer glands. In (B), the arrow points a long and irregular gland and the asterisk demarcates a group of glands that are short and more regular.

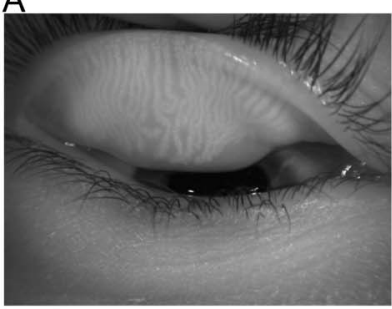

B

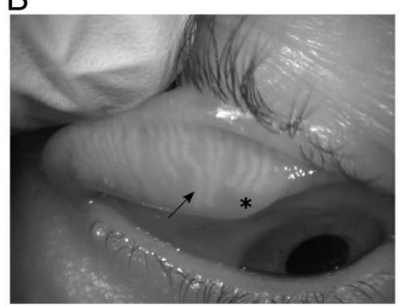

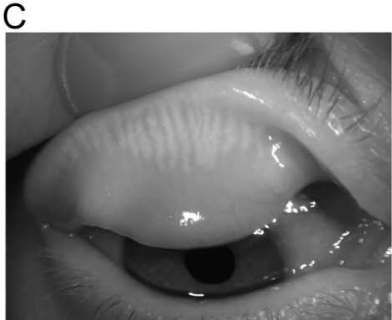

function, ocular surface health, and/or tear film quality. Arita et $\mathrm{al}^{23}$ scored the area of meibomian gland dropout using a subjective 4-grade scale (meiboscore) and found that, for subjects with obstructive MGD, the dropout area significantly correlated with ocular symptoms, lid abnormalities, fluorescein breakup time, and gland expressibility. Using the same criterion, Finis et $\mathrm{al}^{24}$ found lower but still significant correlations between the dropout area and the number of functioning glands in a mixed group of subjects. However, they concluded that the assessment of the dropout area, as a single criterion, is not enough to detect impaired meibomian glands, but it should be interpreted in the context of additional tests. An interesting fact of the work of Finis et al is that $33.3 \%$ of the subjects with no gland loss showed less than 4 functioning glands, suggesting that gland dysfunction also occurs without gland loss. Rico-del-Viejo et $\mathrm{al}^{25}$ found that the dropout area correlated with ocular signs only in subjects with an advanced level of gland loss and reported increased levels of tear osmolarity and ocular surface staining when the dropout area was higher than the $50 \%$.

Some authors have used semiautomated methods, such as ImageJ software, to analyze the dropout area. This semiautomated method still has some interobserver variability because the examiner has to manually define the area of glands. Using such software, Pult and Ride-Pult ${ }^{14}$ found that, for a group of 17 subjects, the area of meibomian gland

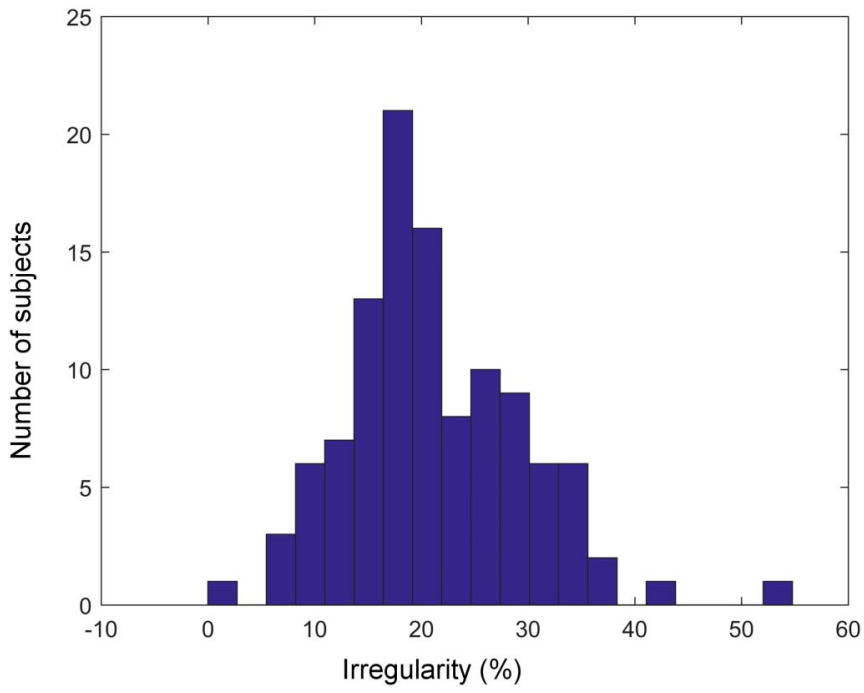

FIGURE 2. Histogram of the distribution of gland irregularity in the group of 110 subjects with dropout area $<32 \%$. dropout significantly correlated with the noninvasive breakup time $(r=-0.46, P=0.032)$ and OSDI $(r=0.89, P=0.001)$. Similarly, a recent study ${ }^{26}$ found a slight but still statistically significant correlation among subjects with MGD between dropout area and OSDI $(r=0.187, P<0.05)$. On the other hand, Eom et $\mathrm{al}^{27}$ did not find any correlation with fluorescein breakup time or the Schirmer test, neither in healthy subjects nor in subjects with MGD. In this study, positive significant correlations were found between dropout area and bulbar conjunctival hyperemia and conjunctival staining, which are indicators of ocular surface irritation and damage that have been associated with abnormal meibum quality ${ }^{28}$ and MGD. ${ }^{29}$ In agreement with previous findings, where an increase in tear volume has been found in patients with MGD,${ }^{30}$ we have found a positive and significant correlation of gland dropout area with tear meniscus height. This is thought to be the reaction of a compensatory reflex in tearing due to increased tear evaporation caused by weakened quality of the tear film lipid layer. ${ }^{31}$

Discrepancies among cited studies may have different causes. First of all, the assessment of the dropout area in previous studies depends on subjective judgments of meibography images, whereas in this study, the assessment is completely objective with no user input. In addition, having a mixed group of healthy and MGD subjects can be a drawback when correlating the dropout area with clinical signs and symptoms because this correlation is expected to be higher in subjects with MGD with associated clinical symptomatology.

Other morphological characteristics of meibomian glands, such as length, width, or shape irregularity, have been suggested to be valuable when assessing MGD. ${ }^{7,8,32}$ However, studies investigating the influence of meibomian gland morphology on meibomian gland function, tear film characteristics, and ocular surface health are scarce. Ban et $\mathrm{al}^{33}$ evaluated the length of the 5 central meibomian glands in a group of healthy subjects using ImageJ. They reported a mean \pm SD gland length of $5.53 \mathrm{~mm} \pm 1.27 \mathrm{~mm}$, which is higher compared with what is reported in the present study. In the work of Ban et al, gland length statistically correlated with gland expressibility $(r=-0.345, P<0.05)$, but not with fluorescein breakup time, contrary to what has been found in this study. Differences in mean length between both studies are understandable because Ban et al measured the 5 glands located at the center of the eyelid (generally the largest imaged section), whereas we measured the mean length of all the exposed glands. 


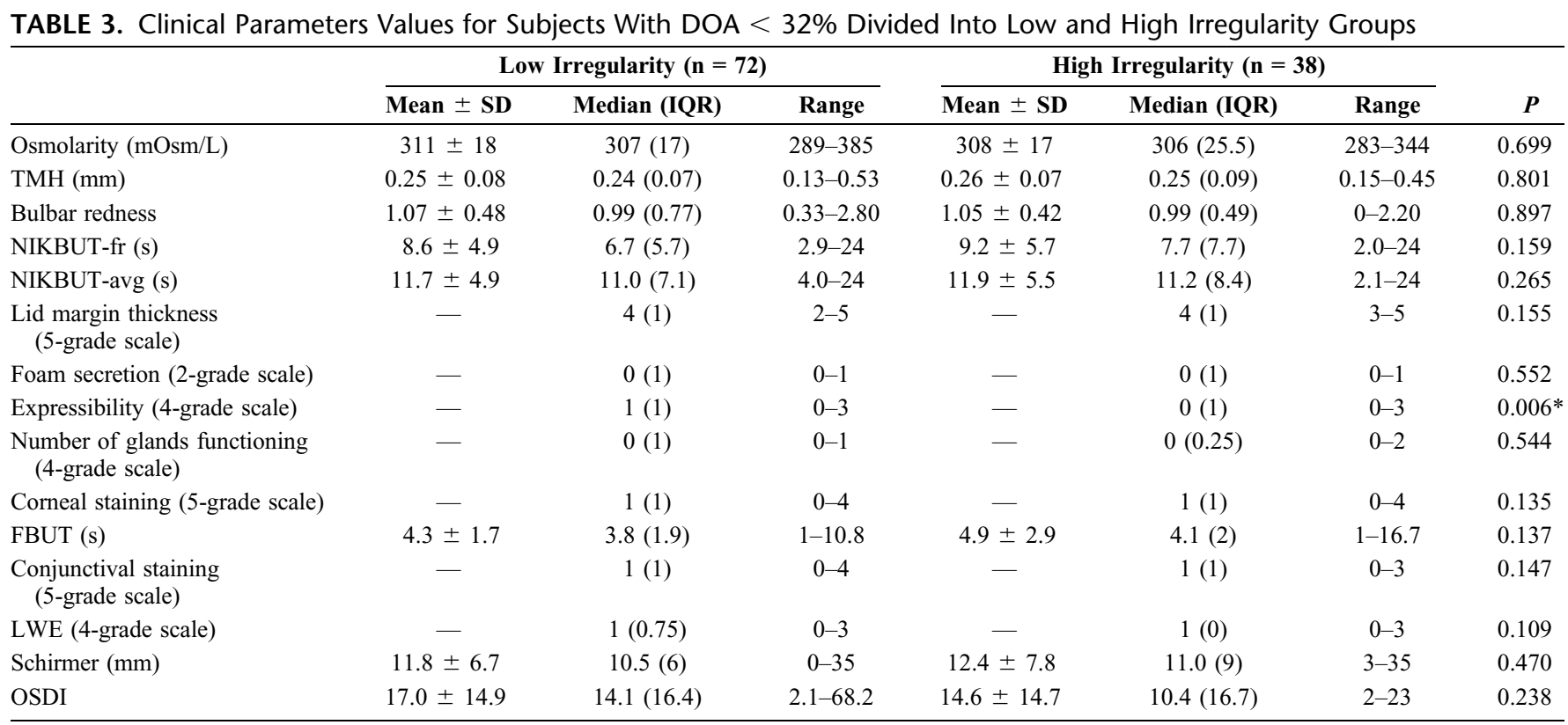

FBUT, fluorescein breakup time; LWE, lid wiper epitheliopathy; NIKBUT-avg, average noninvasive tear film breakup time; NIKBUT-fr, first noninvasive tear film breakup time; $\mathrm{TMH}$, tear meniscus height.

Meibomian gland width was assessed by Pult et al ${ }^{8}$ in a group of 20 random subjects using ImageJ software. The

width of the "worst case gland" was considered to be representative for the overall eyelid condition. A significant correlation between worst case gland width and NIBUT ( $r=$ $-0.761, P<0.001)$ and OSDI $(r=0.469, P=0.033)$ was found. Although in some cases the assessment of the worst gland may be of interest, in general, this is inconclusive because an eyelid with a single impaired meibomian gland is equated with an eyelid that has many impaired glands to the same degree. Finally, they conclude that although gland width adds some value when evaluating meibomian gland condition, the most important factor is the area of gland dropout. In our case, we have found that the mean width of all the glands is weakly but statistically significantly correlated with lid margin thickness, foam secretion, fluorescein breakup time, and conjunctival staining.

Although gland irregularity has been suggested to be a parameter potentially related to meibomian gland function and integrity, 7,23,27,34 to the best of our knowledge, only 1 study has attempted to assess gland irregularity or tortuosity. ${ }^{15}$ However, in that study, irregularity was subjectively assessed as "present" or "absent," and the analysis of how this finding may influence meibomian gland function and the ocular surface condition is lacking. In this study, a quantitative measure of gland irregularity is reported for the first time. Nonetheless, it has been seen that the assessment of gland irregularity only makes sense when glands have a certain minimum length. Therefore, gland irregularity should be a measure subjugated to the percentage of dropout area. When irregularity was assessed in subjects with dropout area $<32 \%$, only gland expressibility showed statistically signif- icant differences between groups of high and low irregularity. Although this difference may seem small (1 grading point in a 4-grade scale), it is considered relevant because it was found in a group of patients with good gland condition. In addition, in the high irregularity group, irregularity positively correlated with the Schirmer test, agreeing with the assumption that compromised gland secretion triggers a compensatory tearing reflex. Hence, it is suggested that gland irregularity could be related to the quality and quantity of the expressed meibum. The lack of other findings related to gland irregularity supports the theory that gland irregularity is not a decisive factor when assessing meibomian glands. We postulate that as long as the gland is not atrophied, it will be functioning although some changes in lipid secretions and composition may occur, leading to an alteration of the tear film properties. Previous studies have demonstrated that changes in the composition of expressed meibum contribute to the development of MGD. ${ }^{35-37}$

One limitation of this study is that only 1 measurement of tear film osmolarity was performed. When using the TearLab Osmolarity System, a minimum of 3 measurements is required to obtain reliable results. ${ }^{38}$ However, because of a logistic reason, this was not possible, entailing a lack of reliability.

The percentage of contact lens wearers in this study is higher than that reported for developed countries. ${ }^{39}$ It is not clear what is the impact of contact lens wear on meibomian gland morphology. ${ }^{40}$ In this study, the objective parameters between contact lens and noncontact lens wearers were not statistically significantly different (for all parameters, Mann-Whitney $U$ test, $P>0.05$ ). In addition, when contact lens wearers were removed from the analysis, the significance of 
correlations did not change. However, if tear film-related signs and symptoms are exacerbated because of the use of contact lenses, that could mask the pure assessment of the effect of meibomian gland morphology on tear film signs and symptoms.

In summary, according to what has been found in this study, isolated morphological characteristics of meibomian glands could not produce a complete assessment of gland function. Some authors have already proved that by integrating different tests, the detection rate of MGD can be improved. ${ }^{8,26}$ Nevertheless, further efforts are needed to ascertain whether a composite score that gathers different meibomian gland morphological features would better describe the influence of meibomian gland function on clinical signs and symptoms, producing a more reliable MGD diagnosis.

\section{REFERENCES}

1. Willcox MDP, Argüeso P, Georgiev GA, et al. TFOS DEWS II tear film report. Ocul Surf. 2017;15:366-403.

2. Nichols KK, Foulks GN, Bron AJ, et al. The international workshop on meibomian gland dysfunction: executive summary. Invest Ophthalmol Vis Sci. 2011;52:1922-1929.

3. Chhadva P, Goldhardt R, Galor A. Meibomian gland disease: the role of gland dysfunction in dry eye disease. Ophthalmology. 2017;124: S20-S26.

4. Arita $\mathrm{R}$, Itoh $\mathrm{K}$, Inoue $\mathrm{K}$, et al. Noncontact infrared meibography to document age-related changes of the meibomian glands in a normal population. Ophthalmol. 2008;115:911-915.

5. Geerling G, Baudouin C, Aragona P, et al. Emerging strategies for the diagnosis and treatment of meibomian gland dysfunction: proceedings of the OCEAN group meeting. Ocul Surf. 2017;15:179-192.

6. Tomlinson A, Bron AJ, Korb DR, et al. The international workshop on meibomian gland dysfunction: report of the diagnosis subcommittee. Invest Ophthalmol Vis Sci. 2011;52:2006-2049.

7. Koh YW, Celik T, Lee HK, et al. Detection of meibomian glands and classification of meibography images. J Biomed Opt. 2012;17:086008.

8. Pult H, Riede-Pult BH, Nichols JJ. Relation between upper and lower lids' meibomian gland morphology, tear film, and dry eye. Optom Vis Sci. 2012;89:E310-E315.

9. Celik T, Lee HK, Petznick A, et al. Bioimage informatics approach to automated meibomian gland analysis in infrared images of meibography. J Optom. 2013;6:194-204.

10. Arita R, Suehiro J, Haraguchi T, et al. Objective image analysis of the meibomian gland area. Br J Ophthalmol. 2014;98:746-755.

11. Koprowski R, Wilczyński S, Olczyk P, et al. A quantitative method for assessing the quality of meibomian glands. Comput Biol Med. 2016;75: $130-138$.

12. Koprowski R, Tian L, Olczyk P. A clinical utility assessment of the automatic measurement method of the quality of meibomian glands. Biomed Eng Online. 2017;16:1-13.

13. Pult H, Nichols JJ. A review of meibography. Optom Vis Sci. 2012;89: E760-E769.

14. Pult H, Riede-Pult BH. Non-contact meibography: keep it simple but effective. Cont Lens Anterior Eye. 2012;35:77-80.

15. Srinivasan $\mathrm{S}$, Menzies $\mathrm{K}$, Sorbara $\mathrm{L}$, et al. Infrared imaging of meibomian gland structure using a novel keratograph. Optom Vis Sci. 2012;89:788-794.

16. Jones RM, Christianson MD, Jacobsen G, et al. Reliability and validity of the ocular surface disease index. Arch Ophthalmol. 2000;118:615-621.

17. Wolffsohn JS, Arita R, Chalmers R, et al. TFOS DEWS II diagnostic methodology report. Ocul Surf. 2017;15:539-574.
18. Bron AJ, Evans VE, Smith JA. Grading of corneal and conjunctival staining in the context of other dry eye tests. Cornea. 2003;22 640-650.

19. Korb DR, Herman JP, Blackie CA, et al. Prevalence of lid wiper epitheliopathy in subjects with dry eye signs and symptoms. Cornea. 2010;29:377-383.

20. Jones LT. The lacrimal secretory system and its treatment. $A m J$ Ophthalmol. 1966;62:47-60

21. Llorens-Quintana C, Rico-del-Viejo L, Syga P, et al. A novel automated approach for infrared-based assessment of meibomian gland morphology. Trans Vis Sci. 2019;8:17-17.

22. Otsu N. A threshold selection method from gray-level histograms. IEEE Trans Syst Man Cybern. 1979;9:62-66.

23. Arita $\mathrm{R}$, Itoh $\mathrm{K}$, Maeda $\mathrm{S}$, et al. Proposed diagnostic criteria for obstructive meibomian gland dysfunction. Ophthalmology. 2009;116: 2058-2063.e1.

24. Finis D, Ackermann P, Pischel N, et al. Evaluation of meibomian gland dysfunction and local distribution of meibomian gland atrophy by noncontact infrared meibography. Curr Eye Res. 2015;40:982-989.

25. Rico-del-viejo L, Benítez-del-castillo JM, Gómez-sanz FJ, et al. The influence of meibomian gland loss on ocular surface clinical parameters. Contact Lens Anterior Eye. 2019 [Epub ahead of print].

26. Giannaccare G, Vigo L, Pellegrini M, et al. Ocular surface workup with automated noninvasive measurements for the diagnosis of meibomian gland dysfunction. Cornea. 2018;37:740-745.

27. Eom Y, Lee JS, Kang SY, et al. Correlation between quantitative measurements of tear film lipid layer thickness and meibomian gland loss in patients with obstructive meibomian gland dysfunction and normal controls. Am J Ophthalmol. 2013;155:1104-1110.e2.

28. Alghamdi YA, Mercado C, McClellan AL, et al. The epidemiology of meibomian gland dysfunction in an elderly population. Cornea. 2016;35: 731-735

29. Shimazaki J, Sakata M, Tsubota K. Ocular surface changes and discomfort in patients with meibomian gland dysfunction. Arch Ophthalmol. 1995;113:1266-1270.

30. Mathers WD. Ocular evaporation in meibomian gland dysfunction and dry eye. Ophthalmology. 1993;100:347-351.

31. Chhadva P, Goldhardt R, Galor A. Meibomian gland disease: the role of gland dysfunction in dry eye disease. Ophthalmology. 2017;124: S20-S26.

32. Robin JB, Jester JV, Nobe J, et al. In vivo transillumination biomicroscopy and photography of meibomian gland dysfunction. A clinical study. Ophthalmology. 1985;92:1423-1426.

33. Ban Y, Ogawa Y, Ibrahim OMA, et al. Morphologic evaluation of meibomian glands in chronic graft-versus-host disease using in vivo laser confocal microscopy. Mol Vis. 2011;17:2533-2543.

34. Robin JB, Nobe J, Suarez E, et al. Meibomian gland evaluation in patients with extended wear soft contact lens deposits. CLAO J. 1986;12: 95-98.

35. McCulley JP, Shine WE. Meibomian gland function and the tear lipid layer. Ocul Surf. 2003;1:97-106.

36. Green-Church KB, Butovich I, Willcox M, et al. The international workshop on meibomian gland dysfunction: report of the subcommittee on tear film lipids and lipid-protein interactions in health and disease. Invest Ophthalmol Vis Sci. 2011;52:1979-1993.

37. Liu S, Richards SM, Lo K, et al. Changes in gene expression in human meibomian gland dysfunction. Investig Opthalmology Vis Sci. 2011;52: $2727-2740$.

38. Szczesna-iskander DH. Contact lens and anterior eye measurement variability of the tearlab osmolarity system. Contact Lens Anterior Eye. 2016:39:353-358

39. Efron N, Morgan PB, Woods CA. International survey of contact lens prescribing for extended wear. Optom Vis Sci. 2012;89:122-129.

40. Arita R, Fukuoka S, Morishige N. Meibomian gland dysfunction and contact lens discomfort. Eye Contact Lens. 2017;43:17-22. 